OPEN ACCESS

Edited by:

Akio Adachi,

Tokushima University, Japan

Reviewed by:

Esmaeil Amiri,

University of North Carolina at Greensboro, United States

Benjamin Lamp,

Vetlmaging, VetCore Facility for Research, University of Veterinary

Medicine, Austria

*Correspondence:

Tatsuhiko Kadowak

tatsuhiko.kadowaki@xitlu.edu.cn

Specialty section:

This article was submitted to

Virology,

a section of the journal

Frontiers in Microbiology

Received: 04 June 2017 Accepted: 02 August 2017 Published: 22 August 2017

Citation:

Wu Y, Dong $X$ and Kadowaki T (2017) Characterization of the Copy Number and Variants of Deformed

Wing Virus (DWV) in the Pairs of Honey Bee Pupa and Infesting Varroa destructor or Tropilaelaps mercedesae.

Front. Microbiol. 8:1558. doi: 10.3389/fmicb.2017.01558

\section{Characterization of the Copy Number and Variants of Deformed Wing Virus (DWV) in the Pairs of Honey Bee Pupa and Infesting Varroa destructor or Tropilaelaps mercedesae}

\author{
Yunfei $W u^{1}$, Xiaofeng Dong ${ }^{2}$ and Tatsuhiko Kadowaki ${ }^{*}$ \\ ${ }^{1}$ Department of Biological Sciences, Xi'an Jiaotong-Liverpool University, Suzhou, China, ${ }^{2}$ School of Life Sciences, Jiangsu \\ Normal University, Xuzhou, China
}

Recent honey bee colony losses, particularly during the winter, have been shown to be associated with the presence of both ectoparasitic mites and Deformed Wing Virus (DWV). Whilst the role of Varroa destructor mites as a viral vector is well established, the role of Tropilaelaps mercedesae mites in viral transmission has not been fully investigated. In this study, we tested the effects that $V$. destructor and T. mercedesae infestation have on fluctuation of the DWV copy number and alteration of the virus variants in honey bees by characterizing individual pupae and their infesting mites. We observed that both mite species were associated with increased viral copy number in honey bee pupae. We found a positive correlation between DWV copy number in pupae and copy number in infesting mites, and the same DWV type A variant was present in either low or high copy number in both honey bee pupae and infesting $V$. destructor. These data also suggest that variant diversity is similar between honey bee pupae and the mites that infest them. These results support a previously proposed hypothesis that DWV suppresses the honey bee immune system when virus copy number reaches a specific threshold, promoting greater replication.

Keywords: honey bee, ectoparasitic mite, deformed wing virus, host-pathogen interaction, viral replication

\section{INTRODUCTION}

Large-scale honey bee colony loss is increasingly being reported across North America and Europe (Goulson et al., 2015). Pollination by honey bees is critical to a functional ecosystem and also production for many crops (Klein et al., 2007; Aizen and Harder, 2009). Prevention of honey bee losses is therefore vitally important to both the apiculture and agriculture industries. Although many potential causes for the decline have been proposed, pathogens and parasites of honey bees, particularly ectoparasitic mites are considered major threats to colony health (Evans and Schwarz, 2011; Goulson et al., 2015). One such mite species, Varroa destructor, is globally distributed (except Australia) and causes both abnormal brood development and brood death in honey bee colonies (Rosenkranz et al., 2010). These effects are elicited through mites feeding on hemolymph and also by spreading honey bee viruses, particularly Deformed Wing Virus (DWV) (de Miranda and Genersch, 2010; Martin et al., 2012). 
In many Asian countries, Apis mellifera colonies are also infested with another ectoparasitic mite, Tropilaelaps mercedesae, and this mite species often co-exist with $V$. destructor in a single colony (Anderson and Morgan, 2007; Luo et al., 2011). $T$. mercedesae has a shorter life cycle than $V$. destructor, without a phoretic phase involving honey bee adults. It also produces a higher number of offspring compared to $V$. destructor (Anderson and Roberts, 2013). However, these two emerging parasites of A. mellifera share many characteristics (Anderson and Roberts, 2013). For example, their reproductive strategies are similar (Sammataro et al., 2000) and both are vectors for DWV (Yue and Genersch, 2005; Dainat et al., 2009; Forsgren et al., 2009). Finally, T. mercedesae and $V$. destructor infestations have similar negative impacts on A. mellifera colonies (Dainat et al., 2012; Khongphinitbunjong et al., 2016; Nazzi and Le Conte, 2016). Although T. mercedesae is currently limited to Asia, the species has the potential to spread and establish in Europe and North America via the global trade in honey bees.

Deformed Wing Virus is phylogenetically classified to the Iflaviridae family in the Picornavirales order (Lanzi et al., 2006). Like other RNA viruses, DWV exists as a quasi-species with three major variants identified (type A, B, and C) (Mordecai et al., 2016a,b). The first characterized DWV and Kakugo virus have now been classified as a type A, while Varroa Destructor Virus-1 (VDV-1) is a type B (Mordecai et al., 2016a). DWV type C has recently been described and is phylogenetically distinct from both A and B types (Mordecai et al., 2016b). Recently, DWV has been successfully reproduced by injecting the in vitro synthesized viral genome RNA to honey bee pupae (Lamp et al., 2016) and the structure of DWV has also been revealed by cryo-EM and X-ray crystallography (Organtini et al., 2017; Škubník et al., 2017).

Previous studies have reported that honey bee colony losses frequently associate with $V$. destructor infestation, as well as increased DWV prevalence and copy number (Highfield et al., 2009; Dainat et al., 2012; Nazzi and Le Conte, 2016). This leads to malformed wings and reduced lifespans during mite infestations (de Miranda and Genersch, 2010; Rosenkranz et al., 2010). The underlying molecular mechanisms for these pathologies as well as the high copy number infection are not yet understood but several different hypotheses have been proposed. These include (1) $V$. destructor induces immune-suppression in the honey bee, stimulating DWV replication (Yang and Cox-Foster, 2005), (2) $V$. destructor selectively amplifies more virulent DWV strains (Martin et al., 2012), (3) DWV suppresses the honey bee immune system when virus copy number reaches a specific threshold, promoting greater replication (Nazzi et al., 2012; Di Prisco et al., 2016), and (4) Direct injection of the mixed variants of DWV to hemolymph of honey bee results in replication of the selective variant to high copy number (Ryabov et al., 2014). However, immune-suppression of honey bee by $V$. destructor infestation has not been observed in other studies (Gregorc et al., 2012; Kuster et al., 2014).

In this study, we compared the effects of $V$. destructor and $T$. mercedesae infestation on DWV prevalence and copy number in honey bee pupae by individually analyzing pupae and infesting mites from the same capped brood cell. Together with sequencing of DWV in paired pupae and mites, we use these data to discuss about the potential mechanisms for the stimulation of DWV replication by $V$. destructor and $T$. mercedesae in honey bees. Many previous studies have analyzed the pooled samples of honey bees and mites, and the results are useful to understand the general degree of DWV infection and identify the dominant variant in the colony. However, our analyses were aimed to understand micro dynamics of DWV population (as well as flow of DWV) between the pairs of honey bee pupa and infesting mite.

\section{MATERIALS AND METHODS}

\section{Sample Collection}

A single A. mellifera colony, sourced from a local beekeeper, was brought to Xi'an Jiaotong Liverpool University in April 2016. Honey bee pupae with purple eyes were collected by opening capped brood cells. Most pupae were free from mites in the period April 20-23 2016, with only a small number infested with $V$. destructor. During May 20-23 2016, we collected further pupae and found that $33 \%$ of the capped brood cells were now infested with $V$. destructor and none of them contained T. mercedesae. When purple eyed-pupae were infested by $V$. destructor, both were sampled simultaneously and stored separately. A second A. mellifera colony infested with $T$. mercedesae was brought in October 2016. Purple eyed-pupae, with or without infesting T. mercedesae, were collected from November 1-8 2016, as described above (50\% of capped brood cells contained T. mercedesae). V. destructor was absent in all of the opened capped brood cells.

\section{RT-PCR Analysis of DWV}

Total RNA was isolated from individual pupae and mites using Total RNA Extraction Reagent (GeneSolution), according to the manufacturer's instructions. Glycogen (1 $\mu \mathrm{g})$ was added to facilitate isopropanol precipitation of the mite RNA samples. Reverse transcription (RT) reactions were carried out using $1 \mu \mathrm{L}$ of total RNA, random primers (TOYOBO), ReverTra Ace (TOYOBO), and RNase Inhibitor (Beyotime). RNase H (Beyotime) was added to digest RNA in RNA/cDNA heteroduplex after cDNA synthesis. These RT products were used for subsequent RT-PCR and qRT-PCR. RT-PCR was conducted to assess DWV infection in sampled pupae and mites using KOD-FX (TOYOBO) and the DWV primer set \#1 (Supplementary Table S1) using the conditions $2 \mathrm{~min}$ at $94^{\circ} \mathrm{C}$, followed by 32 cycles of $10 \mathrm{~s}$ at $98^{\circ} \mathrm{C}, 20 \mathrm{~s}$ at $55^{\circ} \mathrm{C}$, and $30 \mathrm{~s}$ at $68^{\circ} \mathrm{C}$, with a final $3 \mathrm{~min}$ extension at $68^{\circ} \mathrm{C}$. PCR products were analyzed on a $2 \%$ agarose gel. PCR targeting

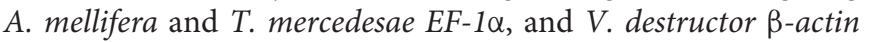
mRNAs (Supplementary Table S1), were used as controls to verify successful RT (Supplementary Figure S1).

\section{qRT-PCR Analysis of DWV Copy Number}

We performed qRT-PCR using a Hieff ${ }^{\mathrm{TM}}{ }_{\circledast}$ qPCR SYBR Green Master Mix (Low Rox Plus, Yesen) and DWV primer set \#2 (Supplementary Table S1) with a QuantStudio5 Real-Time PCR 

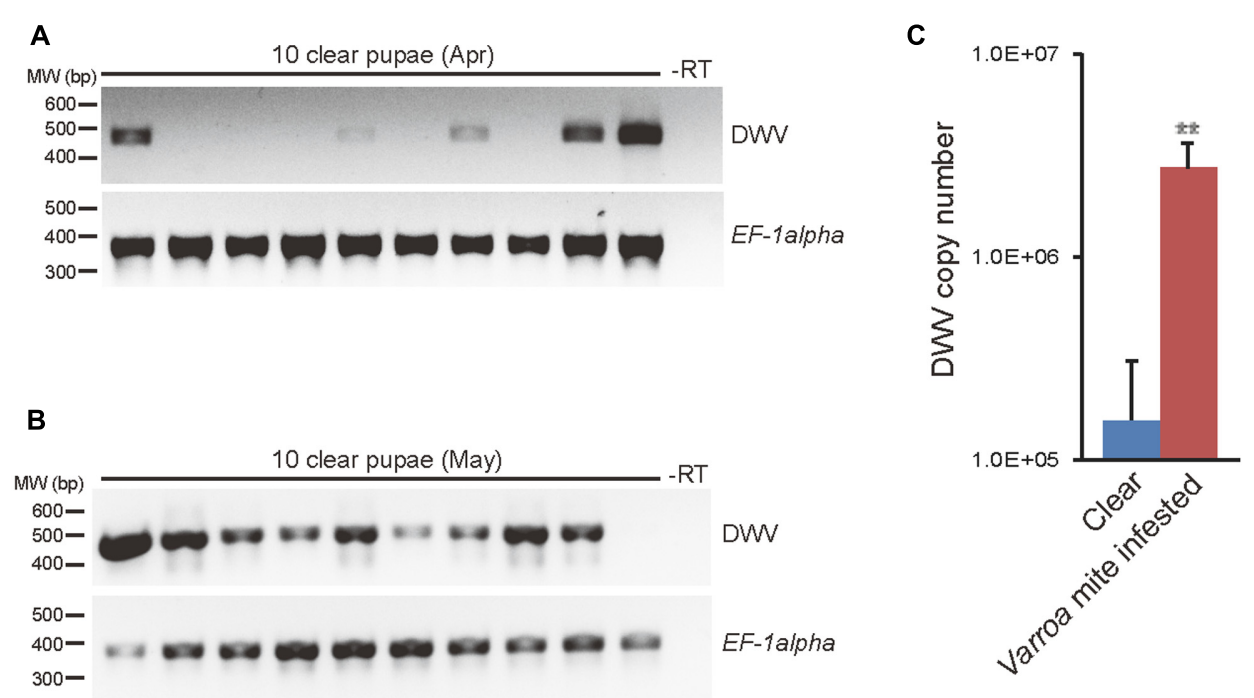

D
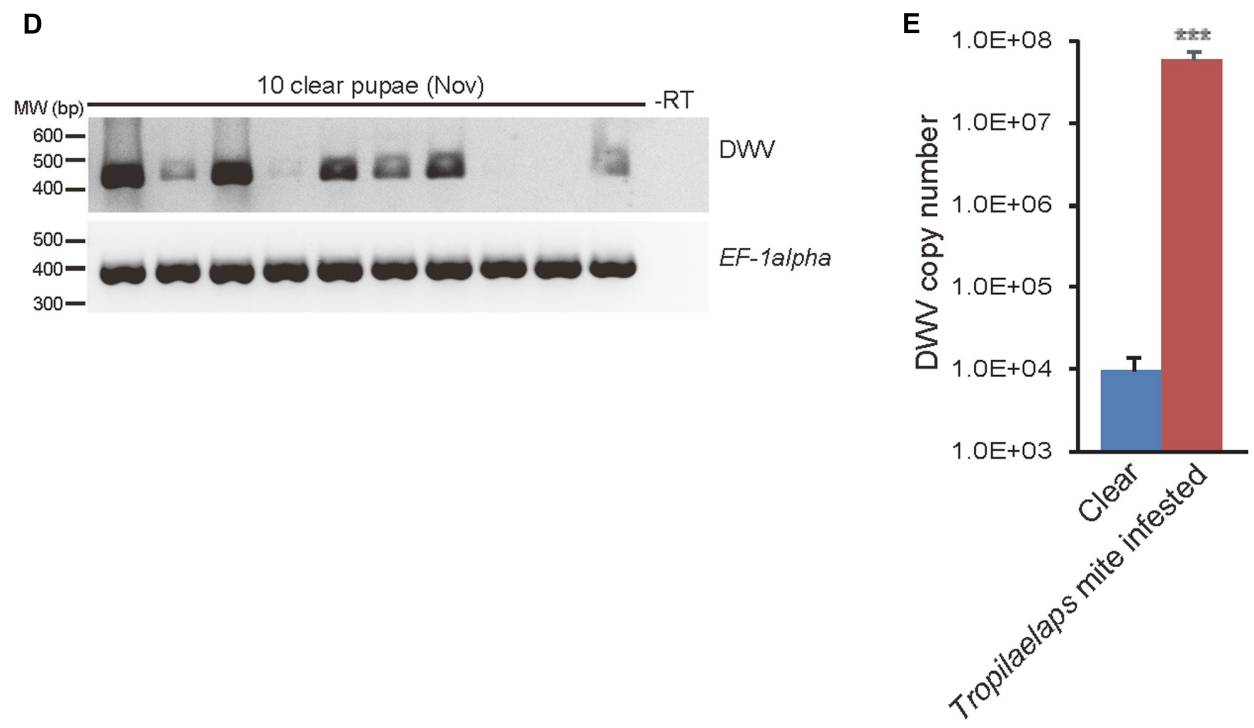

FIGURE 1 | Detection and quantification of DWV in mite-infested and clear honey bee pupae. DWV was detected using RT-PCR in 10 purple eyed-pupae without direct $V$. destructor infestation sampled in (A) April 2016 when the mite infestation was low and in (B) May 2016 when the infestation was high. Honey bee EF-1alpha mRNA was used as an endogenous positive control and water (-RT) used as a negative control. The position of a 400-600 bp DNA molecular weight marker (MW) is shown (left). (C) DWV copy number in purple eyed-pupae from $V$. destructor infested $(n=23)$ or clear $(n=23)$ capped cells was measured by qRT-PCR. The mean value with error bar $( \pm$ SEM) is indicated for each sample. The copy number is higher in the mite infested pupae (**) $(P<0.0065$, two-tailed Welch's $t$-test). (D) DWV was detected by RT-PCR in 10 purple eyed-pupae without direct T. mercedesae infestation sampled in November 2016 when the mite infestation was high. (E) DWV copy number in purple eyed-pupae from T. mercedesae infested ( $n=28)$ or clear $(n=19)$ capped cells was measured by qRT-PCR. The mean value with error bar $( \pm$ SEM) is indicated for each sample. The copy number is higher in the mite infested pupae $(* * *)(P<0.000013$, two-tailed Welch's $t$-test).

System (Thermo Fischer). To perform absolute quantification of DWV RNA, we first prepared standard curves that corresponded to DWV target RNA. Target DNA was prepared by PCR, followed by purification with a AxyPrep ${ }^{\text {TM }}$ PCR cleanup kit (Axygen). DNA concentration was measured using a Nanodrop 2000 spectrophotometer (Thermo Fischer) to calculate copy number using the formula Copy number $=$ DNA concentration $(\mathrm{ng} / \mu \mathrm{L}) \times 6.02 \times 10^{23}($ copies $/ \mathrm{mol}) /$ length $(\mathrm{bp}) \times 6.6 \times 10^{11}$ $(\mathrm{ng} / \mathrm{mol})$, in which $6.6 \times 10^{11} \mathrm{ng} / \mathrm{mol}$ is the average molecular mass of one base pair and $6.022 \times 10^{23}$ copies/mol is Avogadro's number. Linear standard curves were then generated using target DNA of $10^{1}-10^{9}$ copy number per reaction, followed by plotting of $\mathrm{Ct}$ values against log copy number values. After RT, the copy number of target RNA in a sample was estimated using the standard curve. The amount of cDNA added to each reaction was normalized by the $\mathrm{Ct}$ values of either A. mellifera, $V$. destructor, or T. mercedesae $18 S$ $r R N A$. 


\section{Sequencing of RT-PCR Products}

PCR products obtained by RT-PCR were purified and directly sequenced by Sanger sequencing. To sequence the $4 \mathrm{~kb}$ DWV RNA region encoding partial sequence for Lp, helicase, and all of the VP proteins, we performed PCR using DWV primer set \#3 (Supplementary Table S1) using the conditions 2 min at $94{ }^{\circ} \mathrm{C}$, followed by 35 cycles of $10 \mathrm{~s}$ at $98^{\circ} \mathrm{C}, 20 \mathrm{~s}$ at $55^{\circ} \mathrm{C}$, and $3.5 \mathrm{~min}$ at $68^{\circ} \mathrm{C}$, with a final $5 \mathrm{~min}$ extension at $68^{\circ} \mathrm{C}$. These PCR products were sequenced as previously described, and categorized into DWV viral subtypes (A, B, and C).

\section{Construction of Bayesian Phylogenetic Trees}

Prior to phylogenetic analysis, we aligned nucleotide sequences using Mafft (v7.307) (Katoh and Standley, 2013) and then used Gblocks (v0.91b) (Castresana, 2000) to automatically eliminate divergent regions or gaps. The best fit nucleotide substitution models were determined for the alignments by Jmodeltest (v2.1.10) with parameters set to "-g 4 -i -f -AIC -BIC -a" (Darriba et al., 2012). Mrbayes (v3.2.3) (Ronquist and Huelsenbeck, 2003) was run with the parameters set to $1,000,000$ (1 sample/100 generations) until split frequencies were below 0.01 . The first $25 \%$ of samples for each run were designated as the burn-in. DWV type A strain (NC_004830.2), DWV type B strain (NC_006494.1), DWV type C strain (CEND01000001.1), and Sacbrood virus (NC_002066.1) were used as references.

\section{RESULTS}

\section{Effects of Infestation by Two Mite Species on DWV Infection in Honey Bee Pupae}

In April 2016, we collected purple eyed-honey bee pupae (6-8 days after capping) from a single colony. In this initial screen, there were almost no mites but approximately $61 \%$ (11 out of 18) of the honey bee pupae were infected with DWV when analyzed using RT-PCR (Figure 1A for analysis of 10 pupae).

In a second collection from the same colony in May 2016, we found that $V$. destructor was more abundant and $94 \%$ (17 out of 18) of pupae without infesting mites in the capped brood cells were DWV positive (Figure 1B for analysis of 10 pupae) $(P<0.041$; two-tailed Fisher's exact test). We also examined pupae from capped brood cells that were infested with $V$. destructor and found that $100 \%$ were positive for DWV infection (30 out of 30). We compared the copy number of DWV from pupae in mite infested cells to pupae without direct mite infestation. As shown in Figure 1C, mite infested pupae had a higher DWV copy number compared to those without infestation.

In November 2016, we also collected purple eyed-honey bee pupae from a colony infested with T. mercedesae. In this colony, $65 \%$ (13 out of 20) of pupae in capped brood cells without infesting mites were positive for DWV (Figure 1D for analyzing 10 pupae). We again collected pupae in capped brood cells infested by T. mercedesae (100\% positive for DWV, 31 pupae) and

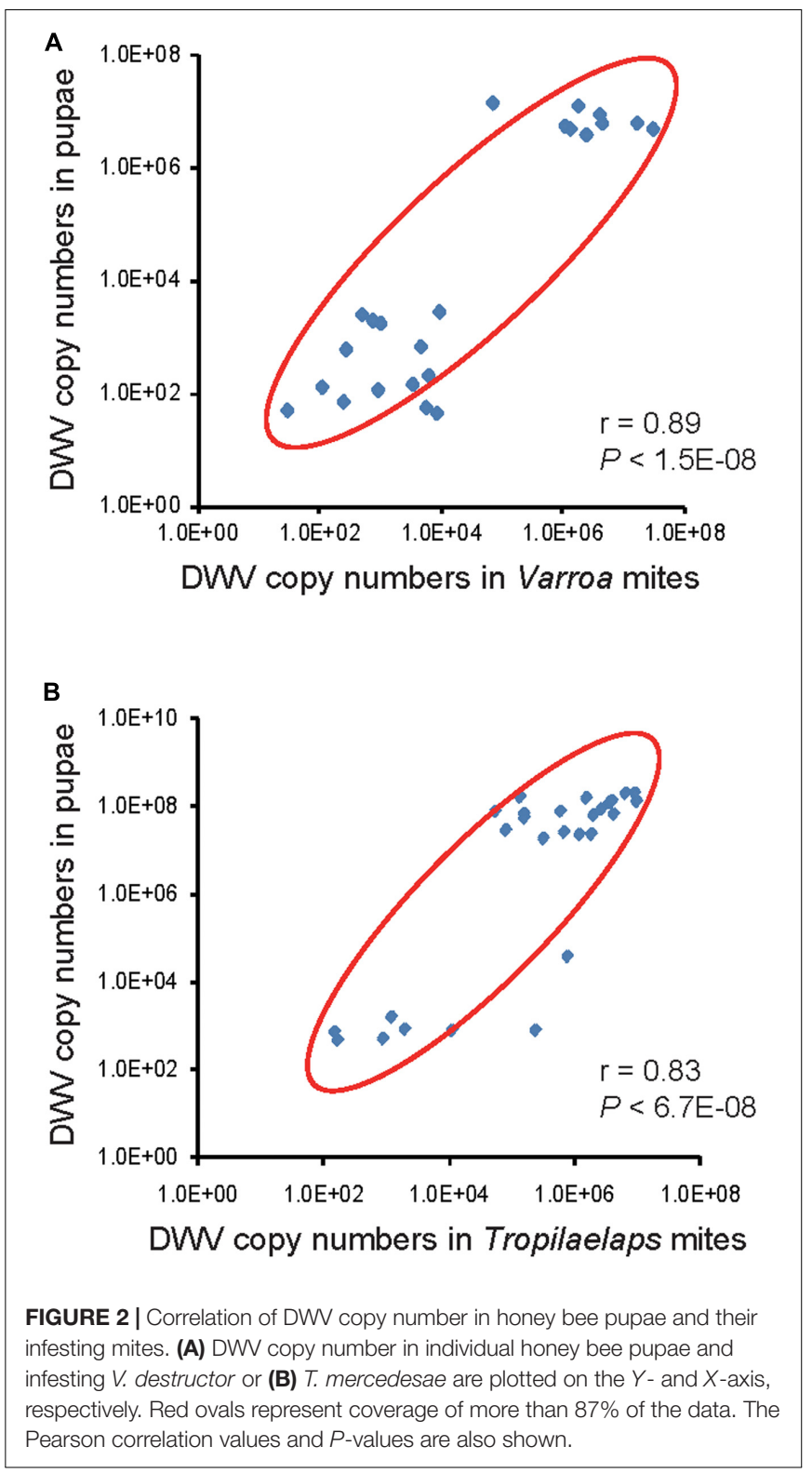

compared their DWV copy number to pupae without direct mite infestation. As shown in Figure 1E, mite infested pupae had a DWV higher copy number than those without infestation.

\section{DWV Copy Number between Honey Bee Pupae and Infesting Mites}

We next measured and compared DWV copy number in 23 honey bee pupa/infesting Varroa mite pairs isolated from the same capped brood cells. We found a linear correlation between DWV copy number in the pupa and infesting mite. Specifically, we observed that the samples grouped into two clusters that were either low or high DWV copy number (Figure 2A). There were more pairs of pupa/mite in the cluster with low copy number of DWV. We also performed the same analysis with 28 honey bee pupa/T. mercedesae capped brood cell pairs; a linear correlation 
and the presence of two copy number clusters was also found in this mite species (Figure 2B). In contrast to $V$. destructor above, there were more pairs of pupa/mite in the cluster with high copy number of DWV in case of T. mercedesae infestation.

\section{DWV Variants and Types Found in Honey Bee Pupae and Mites}

In order to determine the strains of DWV isolates in our study, we directly sequenced RT-PCR products containing the partial sequences for VP2 and VP1, and full sequence of VP4, from 24 honey bee pupa/ $V$. destructor pairs isolated from matched capped brood cells.

Examination of the Sanger sequencing electrograms demonstrated that both pupae and mites could be infected by either a single (Figure 3A) or multiple DWV variants (Figure 3B). For example, in the sequence shown in Figure 3A, single peaks are present at all positions in both forward and reverse sequencing reactions. However, in other samples, multiple peaks are present at equivalent positions in both forward and reverse sequencing reactions (Figure 3B), demonstrating that these multiple peaks are not sequencing artifacts. Nevertheless, it is still possible that deep transcriptome sequencing may reveal the presence of very low prevalence variant(s) in infected pupae or mites that appear infected with a single variant. In our analysis, we found that eight pairs of pupa and Varroa mite shared infection by a single DWV variant, although the variant observed differed between pairs. There were three pairs in which pupae were infected by a single variant and the mites infected by multiple variants. Conversely, there were three pairs in which pupae were infected by multiple variants and the mites a single variant. Finally, the remaining 10 pupa and mite pairs were infected by multiple variants in each (Table 1).

We also analyzed DWV sequences in 17 honey bee pupa/T. mercedesae pairs. We found that two pairs of pupa and
TABLE 1 | Profile of DWV infection in the honey bee pupae and the infesting Varroa mites.

\begin{tabular}{|c|c|c|}
\hline & $\begin{array}{l}\text { Single variant infection in } \\
\text { honey bee pupa }\end{array}$ & $\begin{array}{l}\text { Multiple variants infection } \\
\text { in honey bee pupa }\end{array}$ \\
\hline $\begin{array}{l}\text { Single variant } \\
\text { infection in the } \\
\text { infesting Varroa } \\
\text { mite }\end{array}$ & $7,10,12,17,20,24,28,30$ & $6,18,23$ \\
\hline $\begin{array}{l}\text { Multiple variants } \\
\text { infection in the } \\
\text { infesting Varroa } \\
\text { mite }\end{array}$ & $14,27,29$ & $\begin{array}{l}1,2,5,8,9,13,15,16,22, \\
25\end{array}$ \\
\hline
\end{tabular}

The pupa/mite pairs in the clusters with high and low copy numbers of DWW in Figure 2 are indicated by red and blue letters, respectively. The pairs with black letters were not analyzed in Figure 2.

mite shared infection by single DWV variants, although the viral variant again varied between the pairs. There was a single pupa and mite pair in which the pupa was infected by a single variant and the mites infected with multiple variants. There was also one pair in which the pupa was infected by multiple variants and the mites infected by a single variant. The remaining 13 pairs were infected by multiple variants in both pupae and mites (Table 2).

Phylogenetic relationships estimated using these sequence (Accession number: MF134306-MF134370), and published DWV type A, B, and C sequences, indicated that all the DWV isolates in our study were more related to DWV type A rather than types B or C (Figures 4A,B). Because most posterior probabilities in the trees were less than 1, we additionally sequenced a $4 \mathrm{~kb}$ region of the viral genome containing part of the Lp sequence, all of the VP genes, and part of the helicase gene, from 14 representative samples ( $V$. destructor, T. mercedesae, mite-infested pupae, and infestation-free pupae) in total. A phylogenetic tree was then estimated from these data (Accession number: MF134371-MF134383). As shown in

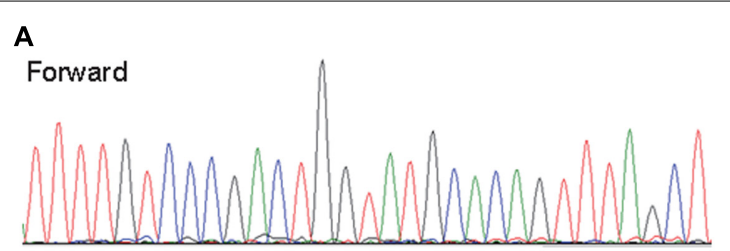

Reverse

B

Forward

Reverse
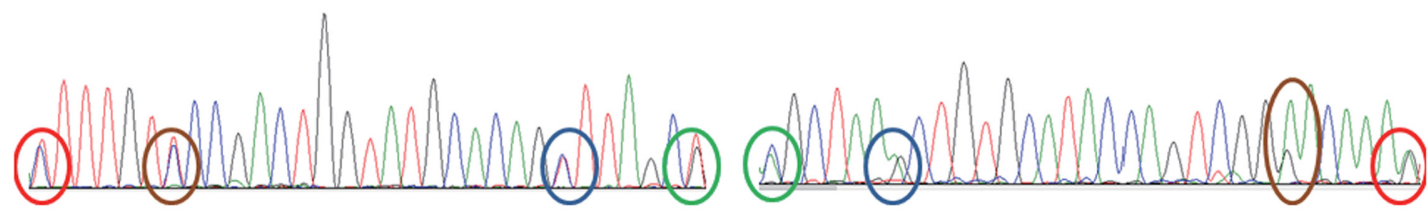

FIGURE 3 | Infections of single and multiple DWV variants in honey bee pupae and infesting mites. Representative Sanger sequencing electrograms (both forward and reverse) of RT-PCR products reveal both single variant and multiple DWV variants infections in honey bee pupae and infesting $V$. destructor or T. mercedesae. (A) Only single peaks are present at all positions of this sample but (B) two peaks are present at four positions in the second sample (marked by red, brown, blue, and green ovals in both forward and reverse sequences). 
TABLE 2 | Profile of DWV infection in the honey bee pupae and the infesting Tropilaelaps mites.

\begin{tabular}{|c|c|c|}
\hline & $\begin{array}{l}\text { Single variant infection } \\
\text { in honey bee pupa }\end{array}$ & $\begin{array}{l}\text { Multiple variants infection } \\
\text { in honey bee pupa }\end{array}$ \\
\hline $\begin{array}{l}\text { Single variant } \\
\text { infection in the } \\
\text { infesting Tropilaelaps } \\
\text { mite }\end{array}$ & 10,13 & 28 \\
\hline $\begin{array}{l}\text { Multiple variants } \\
\text { infection in the } \\
\text { infesting Tropilaelaps } \\
\text { mite }\end{array}$ & 29 & $\begin{array}{l}1,4,5,7,14,15,16,17,18 \\
19,23,26,27,30\end{array}$ \\
\hline
\end{tabular}

The pupa/mite pairs in the clusters with high and low copy numbers of DWV in Figure 2 are indicated by red and blue letters, respectively. The pair 14 (black) was not analyzed in Figure 2.

Figure 5, all isolates are distinct from DWV types B and C and cluster together with type A. Although 2C1 outgroups the other isolates, including the type A (Figure 5), the viral genome sequence encoding the entire polyprotein, as well as parts of the $5^{\prime}$ and $3^{\prime}$ UTRs (Accession number: MF036686) shows higher identity to type A (96\%) rather than types B (84\%) or C (80\%).

\section{Association between DWV Variants and Copy Number in Honey Bee Pupae and Infesting Mites}

As shown in Table 1, 19 honey bee pupa and infesting $V$. destructor pairs were previously classified into high and low DWV copy number clusters (Figure 2). Among these, the same DWV variant was present in both the \#10 pupa/mite pair and \#14 pupa (Figure 4). However, the \#10 and \#14 pairs were classified into high and low copy number clusters, respectively (Table 1). A similar pattern was shown in the \#6 mite sample (high copy number) and \#23 mite sample (low copy number). These results demonstrate that the same DWV type A variant can result in either low or high copy number in both honey bee pupae and infesting $V$. destructor. As all low copy number pairs of pupa and infesting $T$. mercedesae were infected by multiple DWV variants (Table 2), a similar characterization was not possible for this species.

\section{DISCUSSION}

\section{V. destructor and T. mercedesae Infestation Increases DWV Copy Number in Honey Bee Pupae}

Our results demonstrate that an increase in the $V$. destructor population of a colony enhances the prevalence of DWV infection in honey bee pupae, even in pupae without direct mite infestation. Furthermore, direct infestation of pupae with either $V$. destructor or $T$. mercedesae dramatically increased DWV copy number of the pupa. These results support previous reports that $V$. destructor and $T$. mercedesae function as vectors for DWV and promote transmission in honey bees (Shen et al., 2005; Yue and Genersch, 2005; Forsgren et al.,

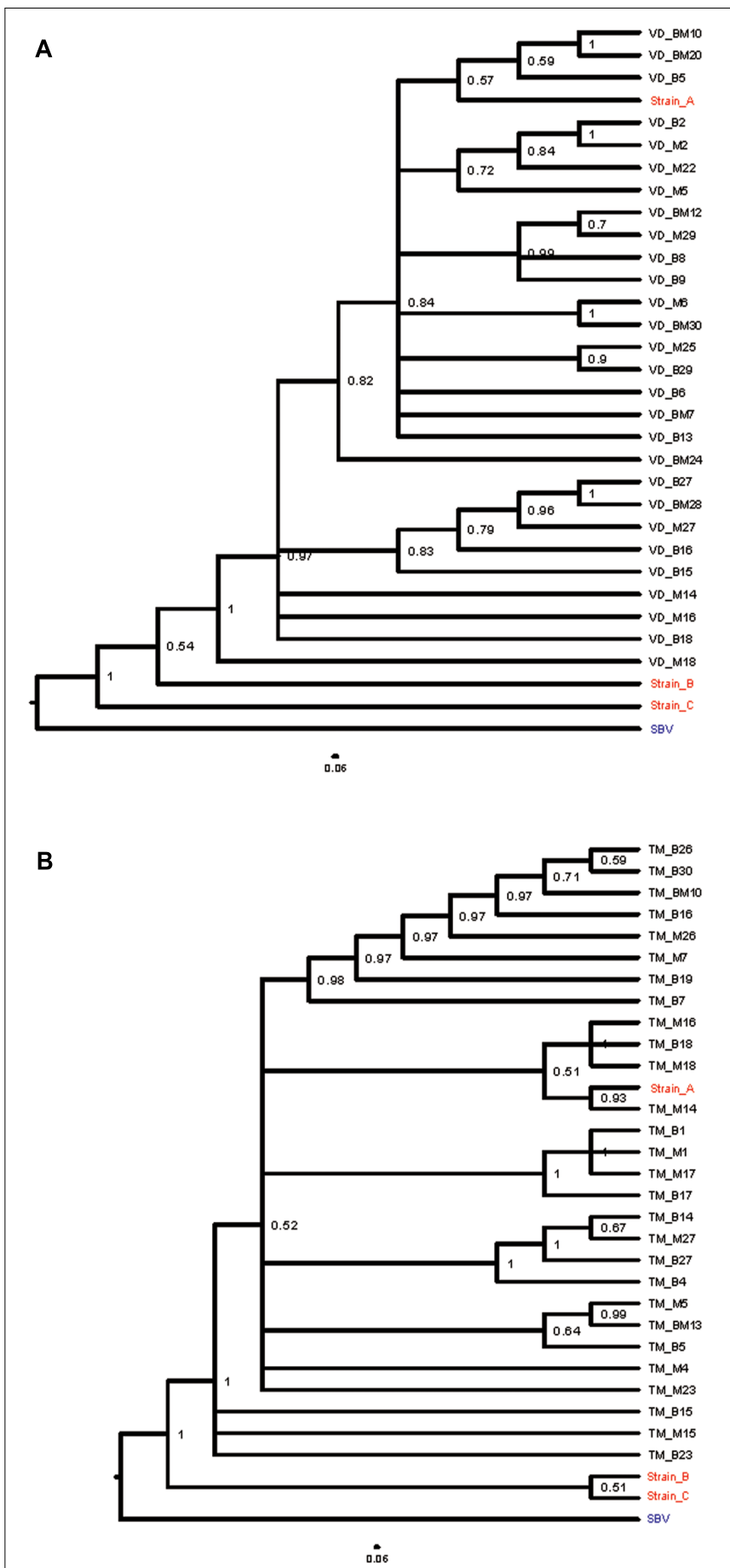

FIGURE 4 | Bayesian phylogenetic trees of DWV isolates based on a 434 nt region encoding viral structural proteins. (A) Bayesian phylogenetic trees of DWV isolates from $V$. destructor infested honey bee pupae (VD_B) and matched mites (VD_M), and (B) T. mercedesae infested honey bee pupae (TM_B) and mites (TM_M), were constructed based on partial VP2 and VP1, and full VP4 sequences. Cases in which a pupa and infesting mites were infected by an identical single DWV variant are indicated as either VD_BM or TM_BM. Posterior probabilities are shown at the corresponding node of each branch. DWV type A, B, and C strains (with red letter) were included for analysis as references and SBV (Sac brood virus) with blue letter was used as an outgroup. 


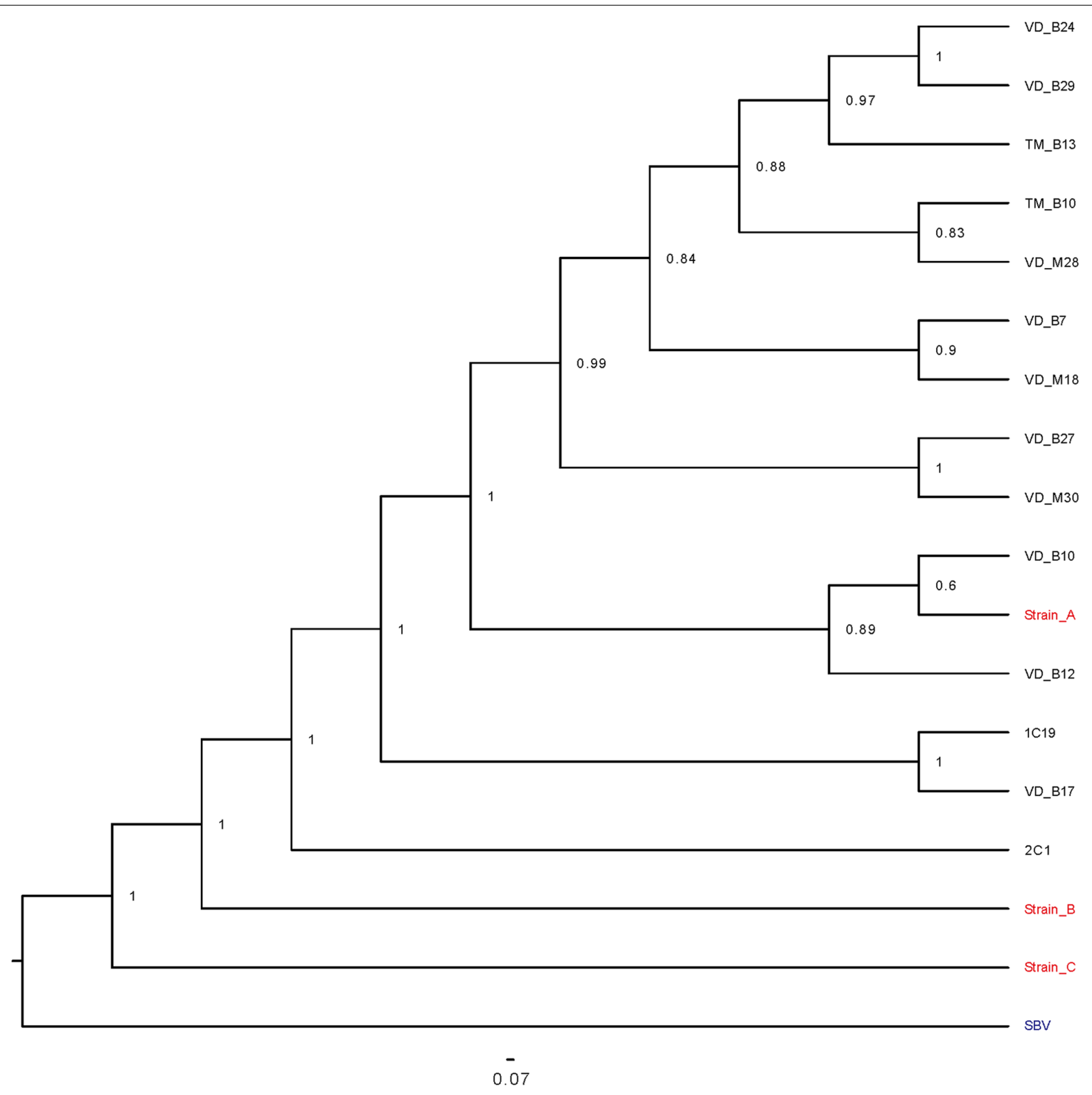

FIGURE 5 | Bayesian phylogenetic tree of representative DWV isolates based on a 3633 nt region encoding viral structural proteins and parts of the $L p$ and helicase proteins. A Bayesian phylogenetic tree of representative DWV isolates was constructed based on partial Lp and helicase sequences, and full VP sequences. $1 C 19$ and $2 \mathrm{C} 1$ were isolated from the mite-free honey bee pupae.

2009). Our study indicates that both mite species are equally effective at increasing DWV copy number in infested honey bee pupae.

These data raise the question as to the source of DWV present in honey bee pupae without direct mite infestation. DWV could be present in larval food (Yue and Genersch, 2005; Singh et al., 2010) and that copy number may increase if honey bee workers have high DWV copy number due to mite infestation. Furthermore, $V$. destructor and T. mercedesae carrying DWV may enter brood cells, feed on hemolymph, but then leave before the cell is capped. Vertical transmission of DWV from the queen may also occur and the mite infestation of drone would enhance this process by producing the semen containing high titer of DWV (Chen et al., 2006; Yue et al., 2007; de Miranda and Fries, 2008).

The same question as to the initial source of DWV also applies to virus found in $V$. destructor and $T$. mercedesae. DWV may again be vertically transmitted from the mother mites and this hypothesis is supported by our recent study demonstrating that $T$. mercedesae males and nymphs have high DWV copy numbers that are the same as females (Dong et al., 2017). In addition, DWV could be horizontally transmitted from infested pupae (for both $V$. destructor 
and T. mercedesae) or adult workers (for $V$. destructor only).

We have confirmed that there is a linear correlation between DWV copy number in honey bee pupae and the mite individual that infests them, supporting previous studies (Forsgren et al., 2009; Kielmanowicz et al., 2015). For both $V$. destructor and T. mercedesae, pupa/mite pairs clustered into two groups containing either low or high DWV copy number. These results suggest that DWV replication in honey bee pupae is positively regulated relative to the amount of DWV introduced by infesting mites. As different DWV variants were found in the honey bee pupa/mite pairs (Tables 1, 2), it seems that DWV does not simply exchange/flow between pupa and mite. We found that both honey bee pupae and mites can be infected by either a single variant or multiple DWV variants before the mite enters the brood cell containing the fifth instar larva. In fact, we found that 19 out of 20 pupae were infected by multiple DWV variants, despite no direct $V$. destructor infestation.

\section{V. destructor and T. mercedesae Mediated Control of DWV Replication in Honey Bee Pupae}

The fact that $V$. destructor infestation increases DWV copy number in honey bees was well established (Shen et al., 2005; Yue and Genersch, 2005) and four major mechanisms have been previously proposed, (1) $V$. destructor induces immune-suppression in honey bees, stimulating DWV replication (Yang and Cox-Foster, 2005), (2) Replicationactive more virulent DWV strains are selected for by $V$. destructor infestation (Martin et al., 2012), (3) DWV induces immune-suppression in honey bees at a threshold copy number (Nazzi et al., 2012; Di Prisco et al., 2016), and (4) Direct injection of the mixed variants of DWV to hemolymph of honey bee results in replication of the selective variant to high copy number (Ryabov et al., 2014). Our results seem to be inconsistent with the first and second proposed mechanisms.

If mite-mediated immune-suppression in honey bees occurs irrespective of DWV copy number in infesting mites, the copy number found in the paired honey bee pupae would not correlate with that in the mites. However, we found a strong correlation in copy number between mites and pupae, suggesting a relationship. Furthermore, even though the DWV isolates in our study were classified as the virulent type A (Mordecai et al., 2016a,b), the same variants were present in low and high copy number clusters in both honey bee pupae and mites. The patterns of DWV infection with either single or multiple variants in honey bee pupae and infesting mites appear to be the same (Tables 1, 2), suggesting that $V$. destructor and $T$. mercedesae may not select for a specific type A variant. This observation is similar to previous report suggesting that a single type of virulent DWV is amplified in honey bee pupae, despite the infesting $V$. destructor contains a diverse population of DWV strains (Ryabov et al., 2014). However, this may not be the case if other types (B and C) co-exist (Mordecai et al., 2016a). Based on several reports, both types $\mathrm{A}$ and $\mathrm{B}$ can be equally virulent (McMahon et al., 2016; Mordecai et al., 2016a). Selection of specific virulent DWV types/variants may depend on the extent of replication in the mites, although the replication mechanism of DWV in honey bees and mites is not yet fully understood.

Our data are most consistent with the third proposed mechanism: that there is immune-suppression at a threshold copy number. This support derives from our observation that DWV replication in honey bee pupae appears to depend on the copy number of DWV introduced by infesting mites. Honey bee pupae may be able to suppress DWV replication if only low levels of virus are introduced by the mite, keeping DWV copy number in the pupae relatively low. Having two major clusters of the pupa/mite pairs with low and high (but not medium) copy number of DWV (Figure 2) also supports this hypothesis. Surprisingly, the same results were basically obtained with two different mite species, suggesting that the common mechanism is likely to operate. If this model is correct, the dominant DWV variant present in the mite should also be the dominate type in the pupa of each high DWV copy number pupa/mite pair. Our study suggests that this is the case for both $V$. destructor and T. mercedesae. For example, the same DWV variant was present in all pupa/ $V$. destructor pairs if they were singly infected, irrespective of copy number. In pair \#6, the single variant present in the mite dominated the multiple variants found in the honey bee pupa. Both pupae and $V$. destructor in the pairs of \#8 and \#16 were infected by multiple DWV variants, although the dominant variant was shared between them (Table 1). The same pattern also occurs in pairs of pupa/T. mercedesae (Table 2). These findings support the hypothesis that DWV replication in honey bee pupae is positively regulated relative to the amount of DWV introduced by infesting mites. This may be due to immune-suppression in the pupae induced by high DWV copy number (Nazzi et al., 2012; Di Prisco et al., 2016). Our results do not fit well to the fourth proposed hypothesis above. Many individuals of $V$. destructor and $T$. mercedesae were infected by multiple variants and most of the mite-infested honey bee pupae were infected by multiple variants at either low or high copy number (Tables 1, 2). This hypothesis may also depend on the copy number of DWV injected as the third proposed mechanism. Our discussion above is based on the results obtained with $23 \mathrm{pupa} / V$. destructor and $29 \mathrm{pupa} / T$. mercedesae pairs, and thus analyzing more pairs in multiple colonies will be necessary to strengthen our arguments.

As the copy number of DWV in $V$. destructor or T. mercedesae is the critical factor determining the extent of DWV replication in infested honey bees, it is important to understand the transmission and replication mechanisms of DWV in the mites as these may differ from those in honey bee. The DWV population is extremely heterogeneous, with many variants and recombinants between different types in both honey bees and mites, even in the single colony. This viral heterogeneity may emerge because there are multiple hosts for the virus (honey bee, $V$. destructor, and T. mercedesae). 
It is thus important to understand how actively the different variants and recombinants replicate in honey bee and mite.

\section{AUTHOR CONTRIBUTIONS}

YW and XD conducted all experiments. YW and TK designed the experiments and wrote the manuscript.

\section{FUNDING}

This work was supported by Jinji Lake Double Hundred Talents Programme and XJTLU Research Development Fund (RDF-1501-25) to TK.

\section{REFERENCES}

Aizen, M. A., and Harder, L. D. (2009). The global stock of domesticated honey bees is growing slower than agricultural demand for pollination. Curr. Biol. 19, 915-918. doi: 10.1016/j.cub.2009.03.071

Anderson, D., and Roberts, J. (2013). Standard methods for Tropilaelaps mites research. J. Apic. Res. 52, 1-16. doi: 10.3896/IBRA.1.52.4.21

Anderson, D. L., and Morgan, M. J. (2007). Genetic and morphological variation of bee-parasitic Tropilaelaps mites (Acari: Laelapidae): new and re-defined species. Exp. Appl. Acarol. 43, 1-24. doi: 10.1007/s10493-007-9103-0

Castresana, J. (2000). Selection of conserved blocks from multiple alignments for their use in phylogenetic analysis. Mol. Biol. Evol. 17, 540-552. doi: 10.1093/ oxfordjournals.molbev.a026334

Chen, Y., Evans, J., and Feldlaufer, M. (2006). Horizontal and vertical transmission of viruses in the honey bee, Apis mellifera. J. Invertebr. Pathol. 92, 152-159. doi: 10.1016/j.jip.2006.03.010

Dainat, B., Evans, J. D., Chen, Y. P., Gauthier, L., and Neumann, P. (2012). Dead or alive: deformed wing virus and Varroa destructor reduce the life span of winter honeybees. Appl. Environ. Microbiol. 78, 981-987. doi: 10.1128/AEM. 06537-11

Dainat, B., Ken, T., Berthoud, H., and Neumann, P. (2009). The ectoparasitic mite Tropilaelaps mercedesae (Acari, Laelapidae) as a vector of honeybee viruses. Insectes Soc. 56, 40-43. doi: 10.1093/gigascience/gix008

Darriba, D., Taboada, G. L., Doallo, R., and Posada, D. (2012). jModelTest 2: more models, new heuristics and parallel computing. Nat. Methods 9:772. doi: $10.1038 /$ nmeth. 2109

de Miranda, J. R., and Fries, I. (2008). Venereal and vertical transmission of deformed wing virus in honeybees (Apis mellifera L.). J. Invertebr. Pathol. 98, 184-189. doi: 10.1016/j.jip.2008.02.004

de Miranda, J. R., and Genersch, E. (2010). Deformed wing virus. J. Invertebr. Pathol. 103(Suppl. 1), S48-S61. doi: 10.1016/j.jip.2009.06.012

Di Prisco, G., Annoscia, D., Margiotta, M., Ferrara, R., Varricchio, P., Zanni, V., et al. (2016). A mutualistic symbiosis between a parasitic mite and a pathogenic virus undermines honey bee immunity and health. Proc. Natl. Acad. Sci. U.S.A. 113, 3203-3208. doi: 10.1073/pnas.1523515113

Dong, X., Armstrong, S. D., Xia, D., Makepeace, B. L., Darby, A. C., and Kadowaki, T. (2017). Draft genome of the honey bee ectoparasitic mite, Tropilaelaps mercedesae, is shaped by the parasitic life history. Gigascience 6, 1-17. doi: 10.1093/gigascience/gix008

Evans, J. D., and Schwarz, R. S. (2011). Bees brought to their knees: microbes affecting honey bee health. Trends Microbiol. 19, 614-620. doi: 10.1016/j.tim. 2011.09.003

Forsgren, E., de Miranda, J. R., Isaksson, M., Wei, S., and Fries, I. (2009). Deformed wing virus associated with Tropilaelaps mercedesae infesting European honey bees (Apis mellifera). Exp. Appl. Acarol. 47, 87-97. doi: 10.1007/s10493-0089204-4

\section{ACKNOWLEDGMENTS}

We thank Jing He, Weile Chen, and Siyun Huang for their contribution to conduct some of the experiments. We are grateful to Dr. Gregory D. Hurst at The University of Liverpool for revising the manuscript.

\section{SUPPLEMENTARY MATERIAL}

The Supplementary Material for this article can be found online at: http://journal.frontiersin.org/article/10.3389/fmicb. 2017.01558/full\#supplementary-material

FIGURE S1 | Original images of agarose gels shown in (A-C). Honey bee EF-1alpha mRNA and DWV were detected by RT-PCR. Water (-RT) was used as the negative control.

Goulson, D., Nicholls, E., Botías, C., and Rotheray, E. L. (2015). Bee declines driven by combined stress from parasites, pesticides, and lack of flowers. Science 347, 1255957. doi: 10.1126/science. 1255957

Gregorc, A., Evans, J. D., Scharf, M., and Ellis, J. D. (2012). Gene expression in honey bee (Apis mellifera) larvae exposed to pesticides and Varroa mites (Varroa destructor). J. Insect Physiol. 58, 1042-1049. doi: 10.1016/j.jinsphys.2012. 03.015

Highfield, A. C., El Nagar, A., Mackinder, L. C., Noël, L. M., Hall, M. J., Martin, S. J., et al. (2009). Deformed wing virus implicated in overwintering honeybee colony losses. Appl. Environ. Microbiol. 75, 7212-7220. doi: 10.1128/AEM.02227-09

Katoh, K., and Standley, D. M. (2013). MAFFT multiple sequence alignment software version 7: improvements in performance and usability. Mol. Biol. Evol. 30, 772-780. doi: 10.1093/molbev/mst010

Khongphinitbunjong, K., Neumann, P., Chantawannakul, P., and Williams, G. R. (2016). The ectoparasitic mite Tropilaelaps mercedesae reduces western honey bee, Apis mellifera, longevity and emergence weight, and promotes Deformed wing virus infections. J. Invertebr. Pathol. 137, 38-42. doi: 10.1016/j.jip.2016. 04.006

Kielmanowicz, M. G., Inberg, A., Lerner, I. M., Golani, Y., Brown, N., Turner, C. L., et al. (2015). Prospective large-scale field study generates predictive model identifying major contributors to colony losses. PLoS Pathog. 11:e1004816. doi: 10.1371/journal.ppat.1004816

Klein, A. M., Vaissière, B. E., Cane, J. H., Steffan-Dewenter, I., Cunningham, S. A., Kremen, C., et al. (2007). Importance of pollinators in changing landscapes for world crops. Proc. Biol. Sci. 274, 303-313. doi: 10.1098/rspb.2006.3721

Kuster, R. D., Boncristiani, H. F., and Rueppell, O. (2014). Immunogene and viral transcript dynamics during parasitic Varroa destructor mite infection of developing honey bee (Apis mellifera) pupae. J. Exp. Biol. 217, 1710-1718. doi: 10.1242/jeb.097766

Lamp, B., Url, A., Seitz, K., Eichhorn, J., Riedel, C., Sinn, L. J., et al. (2016). Construction and rescue of a molecular clone of deformed wing virus (DWV). PLoS ONE 11:e0164639. doi: 10.1371/journal.pone.0164639

Lanzi, G., de Miranda, J. R., Boniotti, M. B., Cameron, C. E., Lavazza, A. Capucci, L., et al. (2006). Molecular and biological characterization of deformed wing virus of honeybees (Apis mellifera L.). J. Virol. 80, 4998-5009. doi: 10.1128/ JVI.80.10.4998-5009.2006

Luo, Q. H., Zhou, T., Dai, P. L., Song, H. L., Wu, Y. Y., and Wang, Q. (2011). Prevalence, intensity and associated factor analysis of Tropilaelaps mercedesae infesting Apis mellifera in China. Exp. Appl. Acarol. 55, 135-146. doi: 10.1007/ s10493-011-9459-z

Martin, S., Highfield, A., Brettell, L., Villalobos, E., Budge, G., Powell, M., et al. (2012). Global honey bee viral landscape altered by a parasitic mite. Science 336, 1304-1306. doi: 10.1126/science.1220941

McMahon, D. P., Natsopoulou, M. E., Doublet, V., Fürst, M., Weging, S., Brown, M. J., et al. (2016). Elevated virulence of an emerging viral genotype as a driver of honeybee loss. Proc. Biol. Sci. 283:20160811. doi: 10.1098/rspb.2016.0811 
Mordecai, G. J., Brettell, L. E., Martin, S. J., Dixon, D., Jones, I. M., and Schroeder, D. C. (2016a). Superinfection exclusion and the long-term survival of honey bees in Varroa-infested colonies. ISME J. 10, 1182-1191. doi: 10.1038/ismej. 2015.186

Mordecai, G. J., Wilfert, L., Martin, S. J., Jones, I. M., and Schroeder, D. C. (2016b). Diversity in a honey bee pathogen: first report of a third master variant of the deformed wing virus quasispecies. ISME J. 10, 1264-1273. doi: 10.1038/ismej. 2015.178

Nazzi, F., Brown, S. P., Annoscia, D., Del Piccolo, F., Di Prisco, G., Varricchio, P., et al. (2012). Synergistic parasite-pathogen interactions mediated by host immunity can drive the collapse of honeybee colonies. PLoS Pathog. 8:e1002735. doi: 10.1371 /journal.ppat.1002735

Nazzi, F., and Le Conte, Y. (2016). Ecology of Varroa destructor, the major ectoparasite of the western honey bee, Apis mellifera. Annu. Rev. Entomol. 61, 417-432. doi: 10.1146/annurev-ento-010715-023731

Organtini, L. J., Shingler, K. L., Ashley, R. E., Capaldi, E. A., Durrani, K., Dryden, K. A., et al. (2017). Honey bee deformed wing virus structures reveal that conformational changes accompany genome release. J. Virol. 91:e01795-16. doi: 10.1128/JVI.01795-16

Ronquist, F., and Huelsenbeck, J. P. (2003). MrBayes 3: Bayesian phylogenetic inference under mixed models. Bioinformatics 19, 1572-1574. doi: 10.1093/ bioinformatics/btg180

Rosenkranz, P., Aumeier, P., and Ziegelmann, B. (2010). Biology and control of Varroa destructor. J. Invertebr. Pathol. 103(Suppl. 1), S96-S119. doi: 10.1016/j. jip.2009.07.016

Ryabov, E. V., Wood, G. R., Fannon, J. M., Moore, J. D., Bull, J. C., Chandler, D., et al. (2014). A virulent strain of deformed wing virus (DWV) of honeybees (Apis mellifera) prevails after Varroa destructor-mediated, or in vitro, transmission. PLoS Pathog. 10:e1004230. doi: 10.1371/journal.ppat.1004230

Sammataro, D., Gerson, U., and Needham, G. (2000). Parasitic mites of honey bees: life history, implications, and impact. Annu. Rev. Entomol. 45, 519-548. doi: 10.1146/annurev.ento.45.1.519
Shen, M., Yang, X., Cox-Foster, D., and Cui, L. (2005). The role of varroa mites in infections of Kashmir bee virus (KBV) and deformed wing virus (DWV) in honey bees. Virology 342, 141-149. doi: 10.1016/j.virol.2005.07.012

Singh, R., Levitt, A. L., Rajotte, E. G., Holmes, E. C., Ostiguy, N., Vanengelsdorp, D., et al. (2010). RNA viruses in hymenopteran pollinators: evidence of inter-Taxa virus transmission via pollen and potential impact on non-Apis hymenopteran species. PLoS ONE 5:e14357. doi: 10.1371/journal.pone.0014357

Škubník, K., Nováček, J., Füzik, T., Přidal, A., Paxton, R. J., and Plevka, P. (2017). Structure of deformed wing virus, a major honey bee pathogen. Proc. Natl. Acad. Sci. U.S.A. 114, 3210-3215. doi: 10.1073/pnas.161569 5114

Yang, X., and Cox-Foster, D. L. (2005). Impact of an ectoparasite on the immunity and pathology of an invertebrate: evidence for host immunosuppression and viral amplification. Proc. Natl. Acad. Sci. U.S.A. 102, 7470-7475. doi: 10.1073/ pnas.0501860102

Yue, C., and Genersch, E. (2005). RT-PCR analysis of deformed wing virus in honeybees (Apis mellifera) and mites (Varroa destructor). J. Gen. Virol. 86, 3419-3424. doi: 10.1099/vir.0.81401-0

Yue, C., Schröder, M., Gisder, S., and Genersch, E. (2007). Vertical-transmission routes for deformed wing virus of honeybees (Apis mellifera). J. Gen. Virol. 88, 2329-2336. doi: 10.1099/vir.0.83101-0

Conflict of Interest Statement: The authors declare that the research was conducted in the absence of any commercial or financial relationships that could be construed as a potential conflict of interest.

Copyright (C) 2017 Wu, Dong and Kadowaki. This is an open-access article distributed under the terms of the Creative Commons Attribution License (CC BY). The use, distribution or reproduction in other forums is permitted, provided the original author(s) or licensor are credited and that the original publication in this journal is cited, in accordance with accepted academic practice. No use, distribution or reproduction is permitted which does not comply with these terms. 